\title{
Modelagem e distribuição potencial de espécies arbóreas relevantes para a dinâmica sociocultural e ecológica do Parque Nacional de Sete Cidades, Piauí, Brasil
}

\author{
Modeling and potential distribution of tree species relevant to the \\ sociocultural and ecological dynamics of the Sete Cidades National Park, \\ Piauí, Brazil
}

\author{
José Américo de Souza Gaia1(iD \\ Bartolomeu Israel de Souza ${ }^{2}$ \\ Reinaldo Farias Paiva de Lucena ${ }^{3}$ \\ Ramon Santos Souza 4 (it) \\ Carolina Lima Bezerra Gaia ${ }^{5}$
}

\section{Palavras-chaves: \\ Cerrado \\ Parque Nacional \\ Ecologia \\ Etnobotânica}

\begin{abstract}
Resumo
A Modelagem de Nicho Ecológico é importante ferramenta para estabelecer predições de distribuição de espécies no espaço geográfico. O Parque Nacional de Sete Cidades, no Piauí, Brasil, é uma Unidade de Conservação Federal de Proteção Integral, com 6.221 hectares. O objetivo desse trabalho foi determinar a distribuição geográfica e avaliar o impacto das variáveis na predição do habitat de adequação atual de algumas espécies arbóreas. Foram selecionadas seis espécies, em função do seu valor ecológico e sociocultural: Hymenaea courbaril L; Himatanthus drasticus (Mart.) Plumel; Parkia platycephala Benth.; Magonia pubescens A.St.-Hil.; Caryocar coriaceum Wittm e Handroanthus impetiginosus (Mart. Ex DC.). Como resultado, Foram apontadas 130 espécies arbóreas, onde foi identificado que: a distribuição potencial de Parkia platycephala se concentrou em $26,19 \mathrm{~km}^{2}$; Himatanthus drasticus teve o nicho potencial ocupando 18,44 $\mathrm{km}^{2}$; Hymenaea courbaril teve área estimada em $16,19 \mathrm{~km}^{2}$; Handroanthus impetiginosus teve área potencial prevista no modelo em 12,01 km²; Caryocar coriaceum abrangeu 15,06 km²; Magonia pubescens foi prevista em 16,66 $\mathrm{km}^{2}$. Observou-se que na escala de paisagem, as variáveis topoclimáticas e de textura da cobertura vegetal apresentaram um modelo com bom desempenho e validade para predizer a distribuição potencial.
\end{abstract}

\section{Keywords: \\ Cerrado \\ National Park \\ Ecology \\ Ethnobotany}

\footnotetext{
1 Universidade Federal da Paraíba, João Pessoa, Paraíba, Brasil. americogaia@hotmail.com

2 Universidade Federal da Paraíba, João Pessoa, Paraíba, Brasil. bartolomeuisrael@gmail.com

${ }^{3}$ Universidade Federal da Paraíba, João Pessoa, Paraíba, Brasil. rlucena@dse.ufpb.br

${ }^{4}$ Universidade Federal da Paraíba, João Pessoa, Paraíba, Brasil. ramonssouza93@gmail.com

${ }^{5}$ Universidade Federal do Acre, Rio Branco, Acre, Brasil. carolina.gaia@hotmail.com
} 
distribution and to evaluate the impact of the variables in the prediction of the habitat of current suitability of some tree species. Six species were selected, according to their ecological and sociocultural value: Hymenaea courbaril L; Himatanthus drasticus (Mart.) Plumel; Parkia platycephala Benth .; Magonia pubescens A.St.-Hil .; Caryocar coriaceum Wittm and Handroanthus impetiginosus (Mart. Ex DC.). As a result, 130 tree species were identified, where it was identified that: the potential distribution of Parkia platycephala was concentrated in $26.19 \mathrm{~km}^{2}$; Himatanthus drasticus had the potential niche occupying $18.44 \mathrm{~km}^{2}$; Hymenaea courbaril had an estimated area of $16.19 \mathrm{~km}^{2}$; Handroanthus impetiginosus had a potential area estimated in the model at $12.01 \mathrm{~km}^{2}$; Caryocar coriaceum covered $15.06 \mathrm{~km}^{2}$; Magonia pubescens was estimated at $16.66 \mathrm{~km}^{2}$. It was observed that in the landscape scale, the topoclimatic and texture variables of the vegetation cover presented a model with good performance and validity to predict the potential distribution.

\section{INTRODUÇÃO}

A Modelagem de Nicho Ecológico (MNE) tornouse um procedimento comum para determinar a amplitude da distribuição preditiva de espécies arbóreas (AUSTIN, 2002; SOBERÓN; PETERSON, 2005). O modelo é importante ferramenta por ser capaz de estabelecer predições de distribuição de espécies no espaço geográfico em diversas linhas de estudos, portanto se converte em ferramenta fundamental, por exemplo, na identificação potencial da presença de espécies raras ou ameaçadas de extinção (GUISAN et al. 2006); escolha de áreas prioritárias para conservação (ARAÚJO et al. 2004); escolha de espécies para recuperação de áreas degradadas e análise do efeito das mudanças climáticas globais sobre a biodiversidade (HEIKKINEN et al. 2007).

Em linhas gerais, a MNE de modelo preditivo de distribuição de espécies, está fundamentada em um tratamento computacional que combina os dados de ocorrência de uma ou mais espécies com variáveis ambientais, construindo assim, uma representação das condições requeridas pelas espécies. (ANDERSON et al. 2003). Alguns algoritmos têm sido aplicados para criar modelos que representam essas condições, projetando sobre um mapa que exibe as áreas atuais e potenciais de ocorrência dessas espécies.

O método prevê a adequabilidade ambiental às espécies em função das variáveis ambientais. Por isto, o algoritmo de Máxima Entropia (Maxent) tem sido amplamente utilizado para modelar esta adequabilidade ambiental para uma espécie ou mais, encontrando a distribuição da máxima entropia, isto é, que é mais espalhada, ou mais próxima do uniforme, sujeita a um conjunto de restrições que representam informações incompletas sobre a distribuição alvo (PHILLIPS, et al. 2006).

$\mathrm{O}$ Maxent pertence à categoria de algoritmos que permitem gerar modelos mesmo sem registros de ausência das espécies, porque gera pseudo-ausências na área de estudo (WISZ et al. 2008). Esta característica é muito importante, principalmente porque para muitas espécies biológicas há poucos registros de ausência disponíveis (SOBERÓN; PETERSON, 2005).

Em função do exposto, o metodo Maxent tem se mostrado com elevada eficiência para modelagem de habitat em estudos que avaliam a capacidade preditiva de diferentes algoritmos (HIJMANS; GRAHAM, 2006; GIBSON et al. 2007; GUISAN et al. 2007).

Esse trabalho tem como objetivo determinar a distribuição geográfica e avaliar o impacto das variáveis na predição do habitat de adequação atual de algumas espécies de relevância ecológico e sociocultural no Parque Nacional de Sete Cidades, Piauí, Brasil.

\section{MATERIAIS E MÉTODOS}

O trabalho foi realizado no Parque Nacional de Sete Cidades (PNSC), no período de 21 de maio a 8 de junho de 2018 , com pesquisa exploratória na área interna da UC e no seu entorno imediato onde temos a zona de amortecimento, especificamente na comunidade rural denominada Cachoeira, localizada no município de Brasileira, Piauí, Brasil, com o objetivo de identificar a ocorrência de espécies arbóreas e sua relevância para uso nessa área.

A coleta de dados foi realizada por uma equipe multidisciplinar de pesquisadores, com a 
participação de 27 moradores da comunidade rural Cachoeria (NUNES, 2019).

$\mathrm{O}$ método empregado na pesquisa de campo no interior do PNSC foi o caminhamento, desenvolvido em 24 horas intercaladas, para obter reconhecimento fitofisionômico na área de estudo e elaboração da lista de indivíduos, bem como a ocorrência atual das espécies e análise dos resultados (FILGUEIRAS et al. 1994). A plotagem dos indivíduos foi realizado com uso do GPS, no formato tradicional, onde longitude e latitude são representados na forma de graus e minutos. Os quadrantes do globo são representados como N/S (norte/sul) e E/W (leste/oeste)

As espécies identificadas ao longo das caminhadas, foram registradas constando nome da família, gênero e espécie, hábitat, hábito e ocorrência. Desse processo foram selecionas seis espécies com base na fitofisionomia da vegetação obtidas nas pesquisas bibliográficas e nos resultados do valor de uso (VU), fornecidos pelas informações geradas pela população consultada (NUNES, 2019).

\section{Caracterização da área de estudo}

O PNSC possui uma área de 6.221 ha apresentando características de biomas diferentes e intercalados, caracterizando uma zona ecotonal entre áreas subúmidas e semiáridas (CASTRO et al. 2013). Foi instituído pelo Decreto Federal n 50.744/1961, localizandose no nordeste do estado do Piauí, distribuído nos municípios de Piracuruca e Brasileira, tendo como principais vias de acesso a BR-222, trecho Piripiri/Fortaleza, e a BR-343, trecho Teresina/Parnaíba (MATOS; FELFILI, 2010), conforme pode ser observado na Figura 1.

No interior do Parque existem várias nascentes que mantêm riachos de regime intermitente: para leste e norte os cursos d'água são afluentes do Rio Piracuruca, para oeste a drenagem alimenta o riacho da Brasileira (afluente do Rio dos Matos), integrantes da subbacia do Longa, com área de $2.842 \mathrm{~km}^{2}$ (MATOS; FELFILI, 2010).

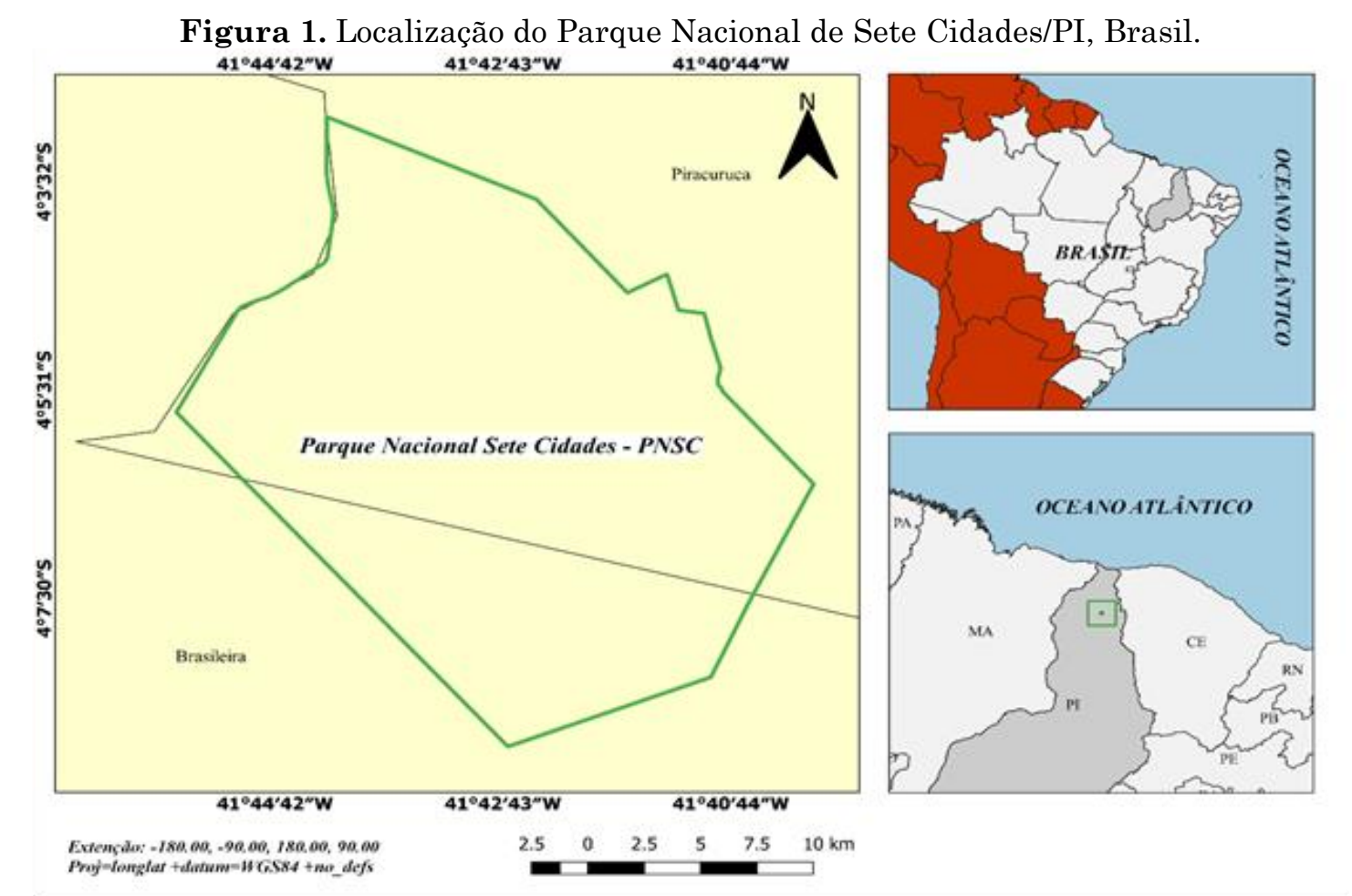

Org.: Autores, 2019.

O clima da área é do tipo Aw (clima tropical, com inverno seco), segundo o sistema classificação de Koppen, apresentando temperatura média anual de $26,5^{\circ} \mathrm{C}$, com máxima de $28,1^{\circ} \mathrm{C}$ e a precipitação média é de $1.200 \mathrm{~mm}$ anuais, semi-árida (MATOS;

\section{FELFILI, 2010).}

O relevo do parque é característico de bacias sedimentares, composto por uma superfície pediplana com altitude entre 100 e 300 m e presença de morros testemunhos isolados em formas cônicas e tabulares. A característica 
principal da vegetação regional é de transição entre os biomas Cerrado e a Caatinga, com predominância de espécies endêmicas do Cerrado seguindo de manchas de campos abertos inundáveis e matas ciliares (BARROS et al. 2014).

Outro estudo sobre Classes de cobertura vegetal do PNSC (OLIVEIRA et al. 2007), permitiram identificar os seguintes tipos de cobertura vegetal: Floresta tropical ombrófila aluvial ocasionalmente inundada, Floresta tropical semidecídua, Floresta aberta latifoliada perenifólia, Cerrado aberto latifoliado perenifólio, Cerrado extremamente xeromórfico e Campo graminóide cespitoso médio.

\section{Dados Ambientais}

O banco de dados ambientais necessário para a modelagem foi constituído do Modelo Digital de Superfície ALOS/World 3D com $30 \mathrm{~m}$ de resolução espacial (AW3D30). A variável declividade, em graus e aspecto, foi calculada através do Google Earth Engine (GEE: $<$ https://code.earthengine.google.com>).

A drenagem fluvial foi gerada a partir do algoritmo Terrain Analysis SAGA GIS, no QGIS 3.4 (QGIS Development Team, 2019).

As formas da superfície e o Índice de Carga de Insolação de Calor Contínuo (CHILI) com resolução espacial de $90 \mathrm{~m}$, foram obtidos em coleções disponibilizadas pela Conservation Science Partners (THEOBALD et al. 2015). Foram realizados mapas de distâncias em relação as formas e da drenagem da superfície no GEE.

Foi gerado o Índice de Vegetação Ajustado ao Solo - SAVI (HUETE, 1988), utilizando bandas RED (sensor Landsat 8) e NIR do Sensor MSI/Sentinel-2 (Multispectral Instrument onboard SENTINEL-2), do dia 03 de março de 2018, com resolução espacial de $10 \mathrm{~m}$, processado na plataforma GEE.

Foram criadas medidas de textura baseadas no SAVI a partir do algoritmo GLCM - Gray Level Coocurrence Matrix (HARALICK et al. 1973; CONNERS et al. 1984). Os descritores de textura computados foram: média, variância, correlação, contraste, dissimilaridade, momento inverso de diferenças (homogeneidade), segundo momento angular e entropia.

Todas as camadas raster foram cortadas para a extensão do PNSC e reamostradas na resolução de $90 \mathrm{~m}$. Para reduzir a multicolinearidade entre as variáveis altamente correlacionadas, estas foram removidas do modelo utilizando a correlação de Pearson $(r \geq$ $0,7)$.

Como resultado desta seleção, destacamos o SAVIcor (correlação) e SAVIvar (variância): aspecto, elevação, declividade, distância do pico/cume (quente), distância do declive superior (quente), distância do declive superior (plano), distância do declive inferior (quente), distância do declive inferior (plano), distância de vales e distância da drenagem.

\section{Modelagem}

Foi realizado a MNE usando o software MaxEnt (padrões da versão $3.3 .3 \mathrm{k}$ ) para construir os modelos de Entropia Máxima, com uso no software "R" (programas ou pacotes do "R" programing que fornece uma grande variedade de técnicas estatísticas (modelagem linear e não linear, testes estatísticos clássicos, análise de série temporal, classificação, agrupamento, ..., R está disponível como Software Livre sob os termos da "Free Software Foundation 's GNU General Public License" em forma de código fonte. Ele é compilado e executado em uma ampla variedade de plataformas UNIX e sistemas semelhantes) com o algoritmo MaxEnt (PHILLIPS et al. 2006), através do pacote dismo (pacote orientado para a modelagem de distribuição de espécies) (HIJMANS et al. 2017). Esse algoritmo demonstrou funcionar melhor com amostras pequenas em relação a outros métodos de modelagem (KUMAR; STOHLGREN, 2009).

O modelo foi rodado usando o método Bootstrap por 100 repetições, onde foram selecionados $75 \%$ dos dados para treinamento e $25 \%$ para teste do modelo (PHILLIPS, 2008) utilizando particionamento $\mathrm{k}$-fold.

$\mathrm{O}$ teste Jackknife foi realizado para determinar a importância das variáveis. Foi usado a área sob a curva AUC ou curva ROC (Área sob a Curva do Operador Receptor), para avaliar o desempenho do modelo. $\mathrm{O}$ valor da AUC varia de 0 a 1 (FIELDING; BELL, 1997). Um valor de AUC até 0,50 indica que o modelo não apresentou melhor desempenho do que a de um modelo aleatório, enquanto o valor de 1,0 indica discriminação perfeita (SWETS, 1988).

Para exibição e análise posterior, prevendo a presença das espécies (0-1), foram reclassificados no QGIS 3.4 com uma referência da classificação proposta por Yang et al. (2013) em cinco classes potenciais: habitat inadequado (0-0.2); habitat pouco adequado $(0,2$ a 0,4$)$; habitat adequado $(0,4$ a 0,6$)$; habitat altamente adequado $(0,6$ a 0,7$)$; 
habitat muito altamente adequado (0,7-1,0). Para cada modelo, calculou-se a área da distribuição, sendo obtidos valores binários de presença (1) e ausência (0), usando um limiar de corte que maximiza a soma entre sensibilidade (verdadeiros positivos) e especificidade (verdadeiros negativos), segundo os dados de teste MaxSS, qual maximiza simultaneamente sensibilidade e especificidade (JIMÉNEZVALVERDE; LOBO, 2007; LIU et al. 2013).

\section{RESULTADOS}

Foram identificadas 130 espécies arbóreas, com os seguintes potenciais de uso: alimento, combustível, construção, forragem, medicinal, ornamental, tecnologia, veneno-abortivo, veterinário e mágico-religioso. As mais expressivas quanto ao (VU) e mais citadas pela comunidade, foram as espécies Pau d'arco $(4,70)$, Pequi $(3,3)$ e Jatobá $(3,11)$, seguidas das espécies Janaguba (0,59), Faveira (0,59) e Tingui (0,22), sendo que Faveira e Janaguba são as de maior ocorrência no PNSC, identificadas durante o caminhamento.

\section{Avaliação de modelos e contribuições de variáveis}

A obtenção dos dados referentes ao número de indivíduos (NI) por espécies, subsidia a construção dos modelos definidos por suas variáveis ambientais e todos os modelos foram estatisticamente mais robustos que o aleatório (AUC $\geq 0.5$ ), (Tabela 1).

Tabela 1. NI e AUC para os modelos Maxent.

\begin{tabular}{lllll}
\hline Família & Espécies & Nome popular & AUC & NI \\
\hline Fabaceae & Parkia platycephala Benth. & Faveira & 0,76 & 963 \\
Apocynaceae & Himatanthus drasticus (Mart.) Plumel & Janaguba & 0,82 & 780 \\
Fabaceae & Hymenaea courbaril L. & Jatobá & 0,83 & 845 \\
Bignoniaceae & Handroanthus impetiginosus (Mart. ex DC.) & Pau d'arco & 0,77 & 241 \\
Caryocaraceae & Caryocar coriaceum Wittm & Pequi & 0,86 & 302 \\
Sapindaceae & Magonia pubescens A.St.-Hil. & Tingui & 0,82 & 304 \\
\hline
\end{tabular}

Org.: do Autor, 2018.

\section{Distribuição Potencial das Espécies}

O mapa preditivo obtido na modelagem mostra que a área de ocorrência potencial de Parkia platycephala Benth. se concentra em 26,19 km², a maior entre as espécies, distribuídos principalmente em áreas próximas ou parcialmente inundadas por riachos. Quando não associados aos cursos hídricos, sua presença está associada às formas de relevo suave-ondulados (Figura 2). As variáveis ambientais e contribuições relativas às previsões dos modelos Maxent, classificadas pela média da importância relativa das espécies, são apresentadas na
Tabela 2.

O nicho potencial de Himatanthus drasticus (Mart.) estendeu-se por $18,44 \mathrm{~km}^{2}$, distribuídos principalmente em mosaicos de manchas florestais, formações campestres ou em áreas inundadas por riachos perenes e temporários (Figura 3).

O nicho potencial de Hymenaea courbaril L, Plumel, foi estimado em $16,19 \mathrm{~km}^{2}$, distribuídos em áreas próximas ou parcialmente inundadas por riachos, principalmente concentrados em ambientes de vegetação arbórea-arbustiva (Figura 4). 
Figura 2. Predição do habitat atual para Parkia platycephala Benth.: (a) mapa binário e pontos de distribuição e (b) distribuição potencial.

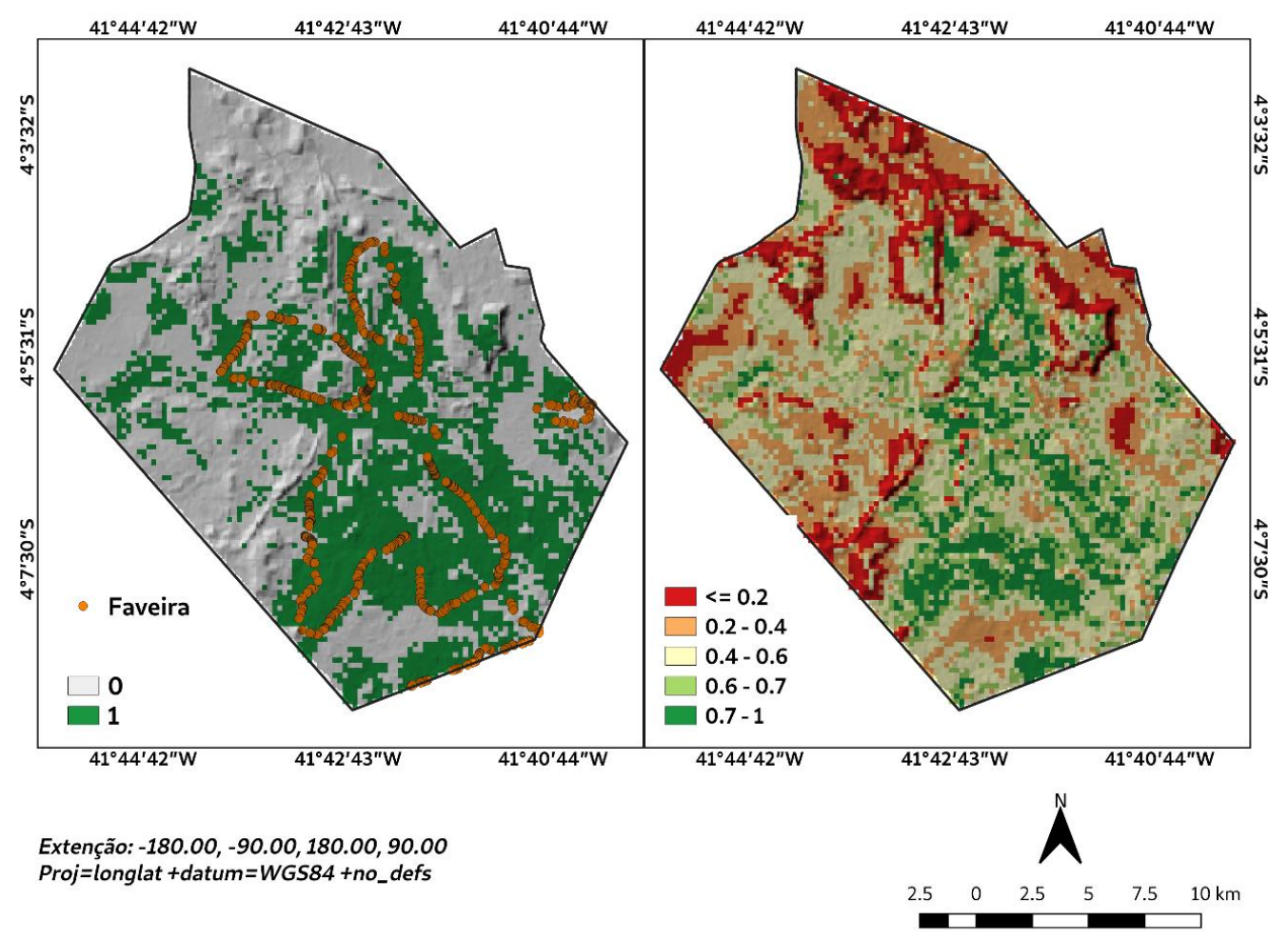

Org.: Autores, 2019.

Figura 3. Predição do habitat atual para Himatanthus drasticus (Mart.): (a) mapa binário e pontos de distribuição e (b) distribuição potencial.

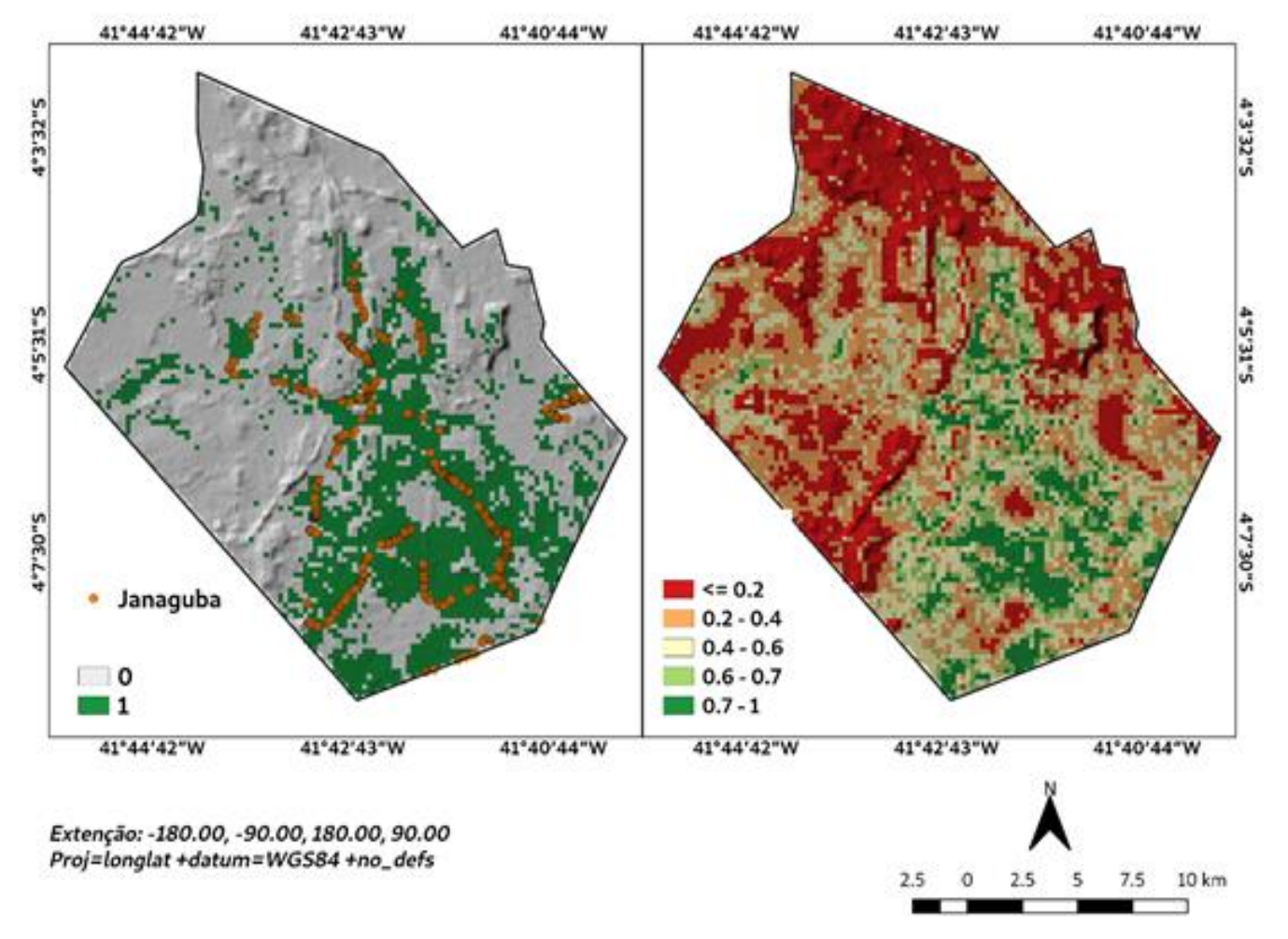

Org.: Autores, 2019. 
Tabela 2. Variáveis ambientais preditoras para as espécies vegetais selecionadas.

\begin{tabular}{|c|c|c|c|c|c|c|}
\hline Variáveis & $\begin{array}{l}\text { Parkia } \\
\text { platycephala } \\
\text { Benth. }\end{array}$ & $\begin{array}{l}\text { Handroanthus } \\
\text { impetiginosus } \\
\text { (Mart. ex DC.) }\end{array}$ & $\begin{array}{l}\text { Himatanthus } \\
\text { drasticus } \\
\text { (Mart.) } \\
\text { Plumel } \\
\end{array}$ & $\begin{array}{l}\text { Hymenaea } \\
\text { courbaril L. }\end{array}$ & $\begin{array}{l}\text { Caryocar } \\
\text { coriaceum } \\
\text { Wittm. }\end{array}$ & $\begin{array}{l}\text { Magonia } \\
\text { pubescens } \\
\text { A.St.-Hil. }\end{array}$ \\
\hline aspecto & 9,10 & $21,28^{* * *}$ & 5,78 & $15,03^{* *}$ & 6,22 & 9,99 \\
\hline elevação & $10,37^{*}$ & 3,52 & $11,59^{*}$ & 9,62 & $14,86^{*}$ & 5,64 \\
\hline SAVIcor & 1,64 & 1,29 & 0,38 & $18,48^{* *}$ & 0,63 & 8,26 \\
\hline SAVIvar & 2,26 & 4,44 & 2,25 & 4,81 & 0,29 & 0,94 \\
\hline declividade & $51,89 * * *$ & $19,71^{* *}$ & $40,46^{* * *}$ & $25,94^{* * *}$ & $19,16^{* *}$ & $37,65^{* * *}$ \\
\hline $\begin{array}{l}\text { distância do } \\
\text { pico/cume } \\
\text { (quente) }\end{array}$ & 8,42 & $12,26^{*}$ & $24,59 * * *$ & 2,10 & $31,58 * * *$ & 5,30 \\
\hline $\begin{array}{l}\text { distância do } \\
\text { declive } \\
\text { superior } \\
\text { (quente) }\end{array}$ & 1,53 & $16,31^{* *}$ & 5,39 & 3,28 & 4,66 & $20,50 * * *$ \\
\hline $\begin{array}{l}\text { distância do } \\
\text { declive } \\
\text { superior } \\
\text { (plano) }\end{array}$ & 6,13 & 0,52 & 3,29 & 7,45 & 1,44 & 1,35 \\
\hline $\begin{array}{l}\text { distância do } \\
\text { declive } \\
\text { inferior } \\
\text { (quente) }\end{array}$ & 4,82 & 6,84 & 3,78 & 4,00 & 2,02 & 3,76 \\
\hline $\begin{array}{l}\text { distância do } \\
\text { declive } \\
\text { inferior } \\
\text { (plano) }\end{array}$ & 1,78 & 9,44 & 1,94 & 0,17 & $12,73^{*}$ & 1,12 \\
\hline $\begin{array}{l}\text { distância de } \\
\text { vales }\end{array}$ & 0,66 & 1,52 & 0,25 & 8,28 & 0,31 & 2,95 \\
\hline $\begin{array}{l}\text { distância da } \\
\text { drenagem }\end{array}$ & 1,41 & 2,87 & 0,29 & 0,84 & 6,09 & 2,55 \\
\hline
\end{tabular}

Previsão: ***importância relativa $>20 \%,{ }^{* *}$ importância relativa $>15 \% \mathrm{e}$ * importância relativa $\left.>10 \%\right)$.

Org.: Autores, 2019. 
Figura 4. Predição do habitat atual para Hymenaea courbaril L. Plumel: (a) mapa binário e pontos de distribuição e (b) distribuição potencial.

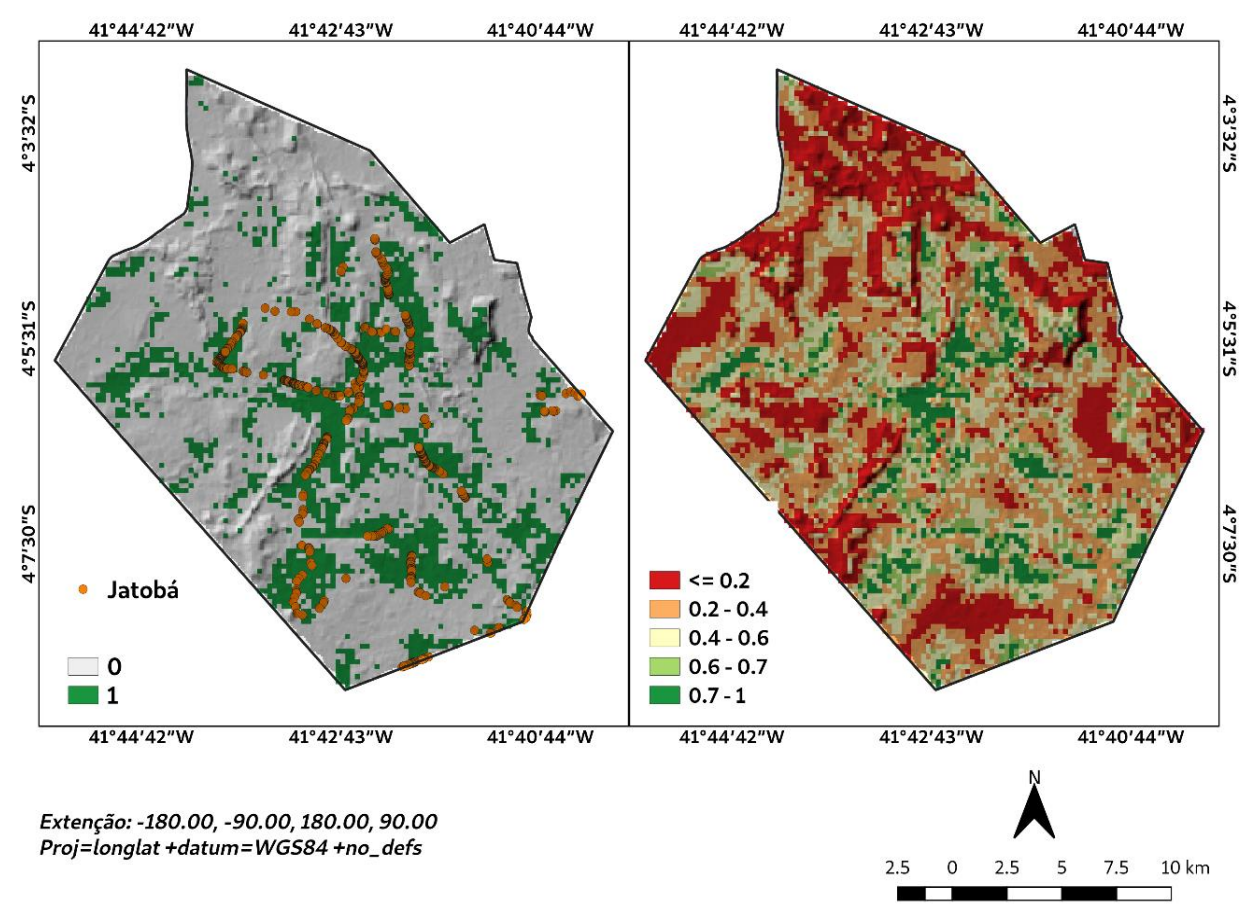

Org.: Autores, 2019.

O modelo previu $12,01 \mathrm{~km}^{2}$ de área potencial atual para Handroanthus impetiginosus (Mart. ex DC.), distribuídos principalmente em locais limítrofes de corpos hídricos e em áreas abertas de cerrado, com características de florestas com processo de sucessão secundária (Figura 5).

Figura 5. Predição do habitat atual para Handroanthus impetiginosus (Mart. Ex DC.): (a) mapa binário e pontos de distribuição e (b) distribuição potencial.

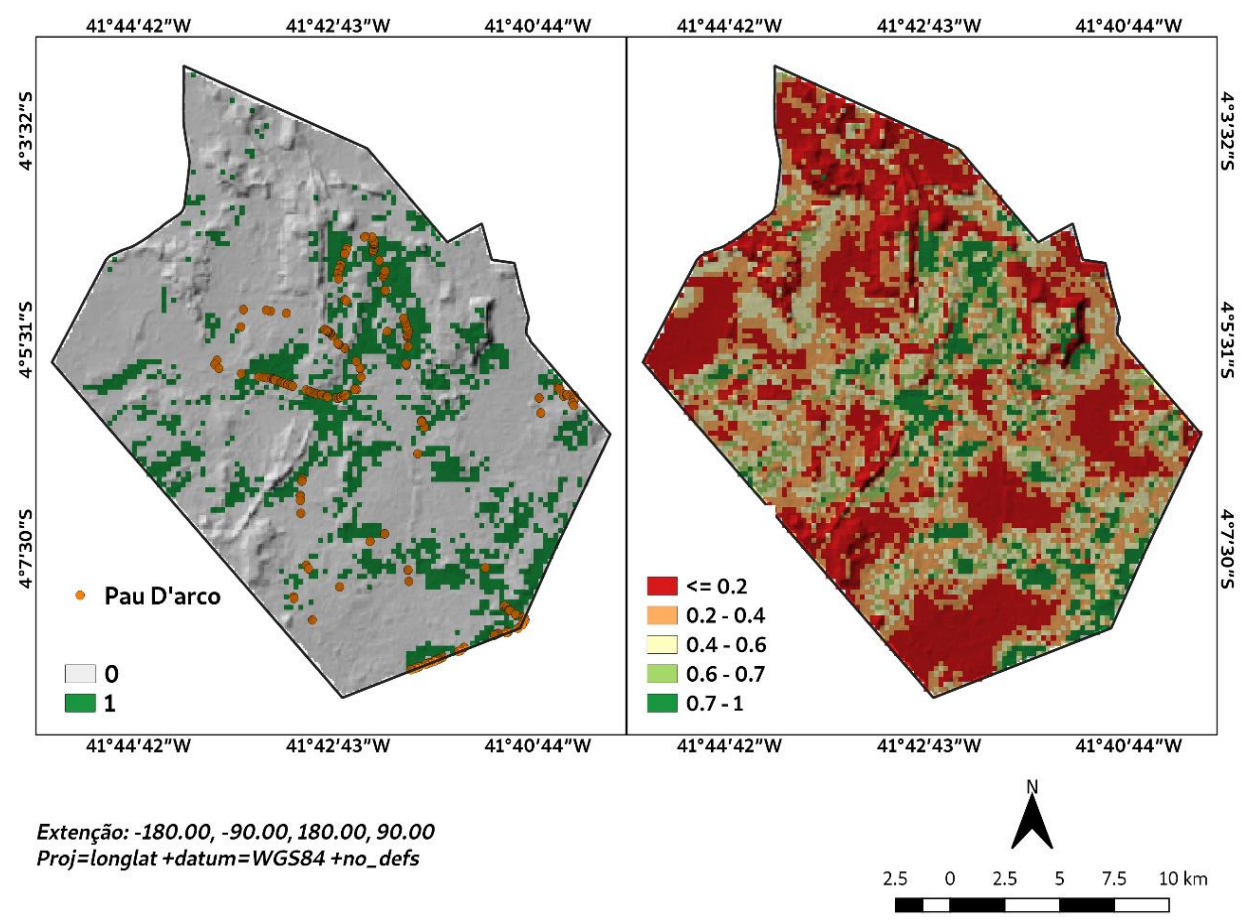

Org.: Autores, 2019. 
O mapa potencial de Caryocar coriaceum Wittm. abrangeu $15,06 \mathrm{~km}^{2}$, caracterizando-se por uma distribuição concentrada nas partes central e sudeste do Parque (Figura 6).

Figura 6. Predição do habitat atual para Caryocar coriaceum Wittm: (a) mapa binário e pontos de distribuição e (b) distribuição potencial.

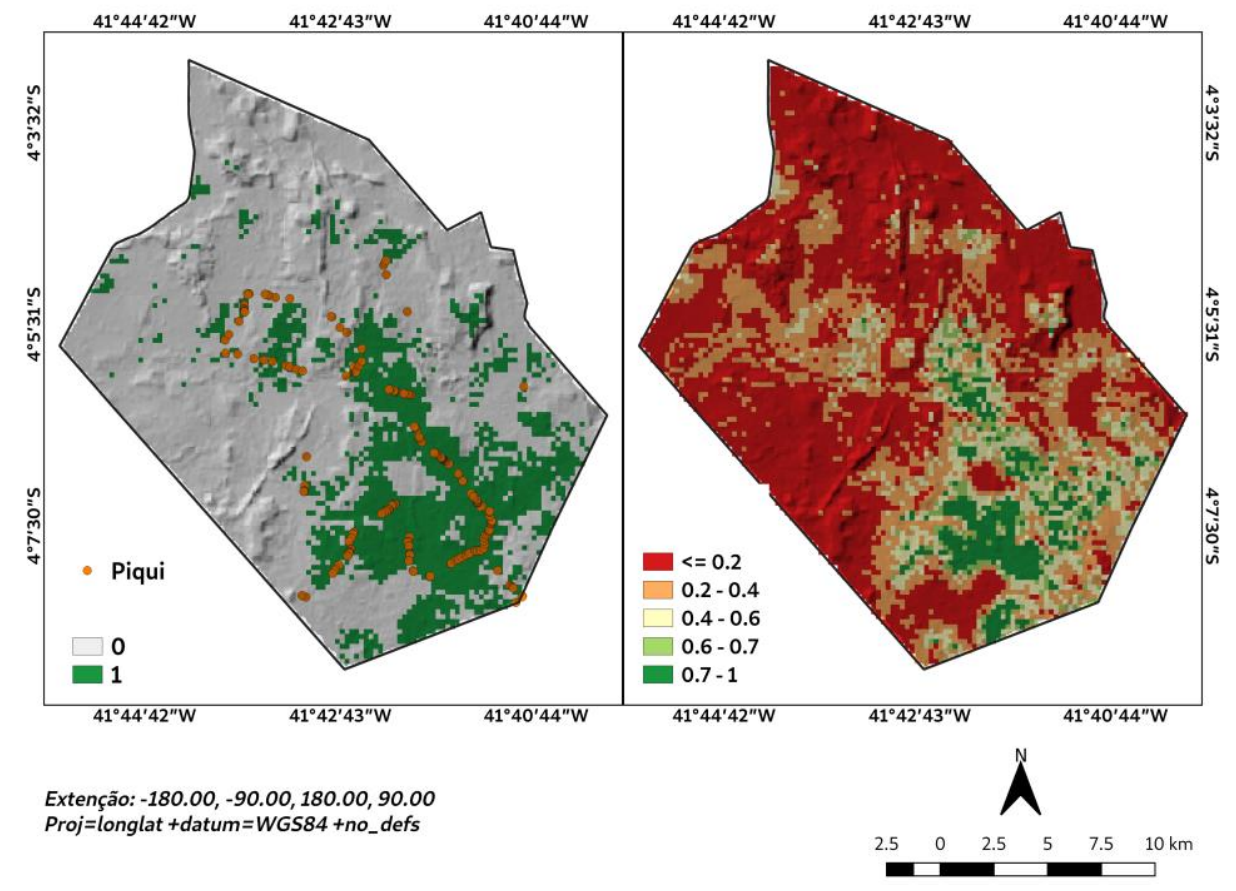

Org.: Autores, 2019.

O mapa potencial para Magonia pubescens A.St.-Hil., abrangeu a segunda maior área entre as espécies, com $16,66 \mathrm{~km}^{2}$, caracterizando-se por uma distribuição em cotas altimetrias mais elevadas, em fragmentos vegetais aglomerados e em cerrados com vegetação mais aberta (Figura 7).

\section{DISCUSSÃO}

\section{Avaliação de modelos e contribuições de variáveis}

As variáveis topoclimáticas como elevação, declividade, aspecto (grau de exposição do relevo), distância do pico/cume (quente), distância do declive superior (quente) e distância do declive inferior (plano) têm um papel importante na distribuição potencial das espécies. Neste contexto, as características nas mudanças fitofisionômica e da biodiversidade de plantas típicas de Cerrado estão relacionadas também as características topográficas que favorecem a diferença do regime hídrico no solo, declividade e rochosidade (LINDOSO et al. 2011).

Assim, os condicionamentos da declividade existentes na superfície do terreno são responsáveis pela dinâmica de transporte de material lixiviado das partes altas para as baixas, condicionado um gradiente de fertilidade, produzido pelo fluxo de água convergente nas superfícies côncavas e divergente nas convexas, por consequência, favorecendo o desenvolvimento das espécies (BOTREL et al. 2002; RESENDE et al. 2007).

Desta forma, a declividade é considerada como um importante fator para a distribuição das espécies Parkia platycephala Benth., Himatanthus drasticus (Mart.) Plumel., Hymenaea courbaril L. e Magonia pubescens A.St.-Hil. 
Figura 7. Predição do habitat atual para Magonia pubescens A.St.-Hil: (a) mapa binário e pontos de distribuição e (b) distribuição potencial.

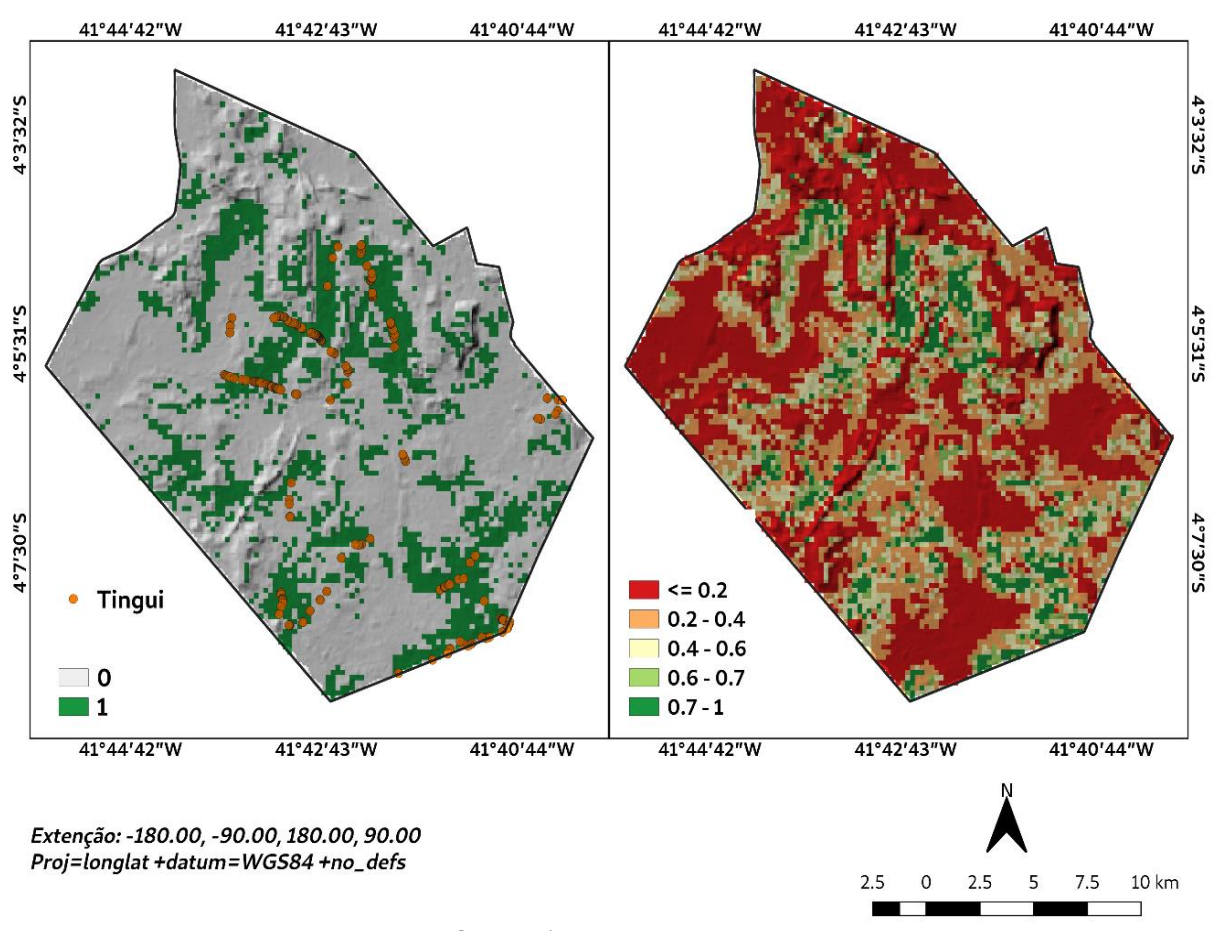

Org.: Autores, 2019.

Já a elevação teve destaque para as espécies Parkia platycephala Benth., Himatanthus drasticus (Mart.) Plumel. E Caryocar coriaceum Wittm. Esta variável pode estar relacionada aos chamados gradientes indiretos, que são aqueles em que a variável não tem efeito direto na fisiologia da planta, mas apresenta correlação com outras como temperatura e precipitação (AUSTIN, 2002).

$\mathrm{O}$ aspecto como fator de exposição da espécie em relação a luminosidade em razão do relevo, também é um fator limitante em relação as distribuições de algumas espécies como Himatanthus drasticus (Mart.) Plumel., Handroanthus impetiginosus (Mart. Ex DC.) Mattos, Caryocar coriaceum Wittm. E Magonia pubescens A.St.-Hil. As variáveis topográficas estão indiretamente relacionadas a produtividade e capacidade de retenção de água no solo, onde as formações florestais como Mata de Galeria e Mata Ciliar estão intimamente ligadas aos córregos e rios (SOLÓRZANO, 2011).

$\mathrm{O}$ aspecto também pode exercer o papel da presença ou ausência circundante da topografia que causa sombreamento, além de controlar a insolação solar, mostrando efeito direto nas temperaturas do solo e taxas de evapotranspiração. Logo, se as taxas de precipitação estão distribuídas uniformemente no local, ocorrendo taxas mais altas de evapotranspiração, dependendo da posição das encostas, resultarão na redução da umidade do solo ou acumulo de água em rochas (PELLETIER; SWETNAM, 2017).

\section{Distribuição Potencial das Espécies}

Mapeou-se nesta pesquisa os habitats das espécies arbóreas Parkia platycephala Benth. Com maior área de adequabilidade, cerca de $22 \%$ do limite total do PNSC.

Himatanthus drasticus (Mart.) Plumel., espécie arbustivo-arbórea típica de Cerrado, teve a segunda maior área de adequabilidade, com $16 \%$ do limite total do parque. Em outro estudo no PNSC, Castro et al. (2010) apontam que o Cerrado típico ocupa a maior área do parque, com $37,6 \%$, caracterizado pela presença de dois extratos, o herbáceo-subarbustivo e o arbustivoarbóreo. Dessa forma, os resultados obtidos no presente trabalho ratificam essa informação.

Foram constatados indivíduos juvenis de Himatanthus drasticus (Mart.) Plumel. Após incêndios recorrentes, o que aparentemente justifica sua distribuição dominante, uma vez que possui capacidade de rebrotar a partir das raízes após a morte das suas partes aéreas pelo fogo (MORO et al. 2011).

Magonia pubescens A.St.-Hil. (Tingui) teve a segunda maior área de adequabilidade, com 
14,44\%, ratificando o trabalho de Carvalho (2016), o qual constatou a presença abundante desta espécie no parque, no estrato arbustivoarbóreo.

Acrescentamos que diferentes intensidades de luz podem provocar modificações na morfologia e fisiologia de plântulas, dependendo do nível de acomodação da espécie (SCALON et al. 2003). Nesse contexto, espécies de Mata de Galeria têm demonstrado melhor crescimento inicial em condições intermediárias de luminosidade (VALADÃO et al. 2014), embora os resultados com relação as condições perfeitas de luminosidade são questionadas. Salgado e Laboriau (1973) verificaram que as sementes do Tingui germinam somente com a presença da luz, sendo classificada como fotoblásticas positivas, por outro lado, Joly et al. (1980) afirmam que as sementes dessa espécie não sofrem influência da luz.

Hymenaea courbaril L. ocupou $14,03 \%$ da área. Essa espécie apresenta relevante importância ecológica, pois é tolerante e resistente às condições desfavoráveis do ambiente onde outras espécies não conseguem sobreviver, estando algumas delas entre as mais presentes em Matas de Galeria, normalmente próximo a mananciais ou ao longo dos rios e riachos, contribuindo para suas proteções.

Dessa forma, é recomendada para reflorestamentos de áreas degradadas e Mata Ciliar, em solos pouco encharcados ou bem drenados (DURIGAN et al. 1990), sendo também considerada promitente para recuperação de áreas contaminadas por metais pesados: cádmio $(\mathrm{Cd})$, chumbo $(\mathrm{Pb})$, cobre $(\mathrm{Cu})$ e zinco $(\mathrm{Zn})$ (MARQUES et al. 1997). Espécie de destaque nas formações savânicas, cobertura vegetal do Cerrado aberto (OLIVEIRA et al. 2007). A espécie é de mata ciliar e campo aberto.

Caryocar coriaceum Wittm. Está distribuída em 13,06\% do território do PNSC, destacando-se no trabalho de Mesquita e Castro (2007) entre as espécies arbóreas de vegetação de Cerrado lato sensu mais presentes no parque.

O Pequi tem como principais agentes de dispersão o homem (catadores), cutia (Dasyprocta aguti), veado (Ozotoceros bezoarticus), mocó (Kerodon upestres), seriema (Cariama cristatae), jacu (Penelope sp.) e até uma espécie de escaravelho (FERREIRA et al. 2009). Há relatos da presença de morcegos (MELO-JUNIOR, et al. 2004) e também de formigas de maior porte (saúvas-cabeça-de-vidro) contribuindo como dispersores da espécie (FERREIRA et al. 2009).

\section{Aspectos do conhecimento local sobre a ocorrência das espécies}

O conhecimento tradicional traz um elo inevitável entre a cultura e a sociedade humana. A formação de uma comunidade, as construções, as teorias das doenças, diagnósticos, tratamento e cura são partes do índice cultural de grupos de pessoas e variam no tempo e no espaço em consonância com a variação cultural (RIVERS, 1924; NASCIMENTO; CÓRDULA, 2016; NASCIMENTO et al. 2014).

O conhecimento local conduz a classificação e conceitos atribuídos ao uso das plantas, requer responsabilidades e respeito, e trata-se de tradições que perpassam gerações. As informações quanto ao conhecimento local, objetivam englobar as relações entre plantas e a cultura humana. Dessa forma, os valores de uso das espécies destacadas na comunidade Cachoeira foram relevantes e subsidiaram a presente pesquisa.

O Jatobá é amplamente utilizado no comércio madeireiro, medicina popular, produção de móveis, construção civil e naval, sendo relevante para os povos indígenas, comunidades tradicionais, agroextrativistas e agriculas, no consumo e como cadeia produtiva, que vai desde a coleta até a comercialização de frutos e derivados. É consumido como alimento e utilizados para fins fitoterápico e artesanato (COSTA, 2011; COSTA et al. 2015).

A Janaguba apresenta importância ecológica muito elevada, considerando que cresce em altitudes que vão de 200 até 1500 , compondo a vegetação do Cerrado e Caatinga, e floresce e frutifica praticamente todo o ano. É uma espécie com muita representatividade na medicina popular, sendo que o látex tem dado respostas relevantes para o tratamento e cura de alguns tipos de câncer (AMARO et. al. 2006).

O Pequi, planta arbórea perene, classificada como frutífera ou oleaginosa, de inúmeras finalidades e aplicações na indústria artesanal, farmacologia e culinária regional, também apresentar potencial de uso para a produção de combustíveis e lubrificantes (GOMES, et al. 2015).

A Faveira destaca-se pelo seu potencial madeireiro, relevante uso como adubação verde e em revegetação de áreas degradadas, na produção de forragem, pois as vagens quando maduras são excelentes na alimentação de ruminantes (ALVES et al. 2007).

O Pau d'arco possui elevada densidade, durabilidade e apresenta alto valor na fabricação 
de móveis e piso, e na aplicação farmacológica, com utilização dos galhos, folhas, tronco, casca e entrecasca com ações anti-inflamatória, analgésica, antibiótica e antineoplásica (LORENZI, 2013).

Da casaca do Tingui pode-se extrair o tanino para extermínio de larvas de Aedes (Stegomyia) aegypti (Linnaeus, 1762) e do carrapato hipicephalus microplus Canestrini (SILVA et al. 2004). É também citado como capaz de levar ao óbito, se a raízes forem ingerida em doses altas e na forma de decocção (BRANDÃO et al. 2002).

\section{CONSIDERAÇÕES FINAIS}

As espécies estudadas na escala de paisagem, quanto as variáveis topoclimáticas e de textura da cobertura vegetal apresentaram um modelo com bom desempenho e validade para predizer a distribuição potencial das espécies arbóreas estudadas.

Sobre os mapas binários de predição do habitat atual das espécies vegetais destacadas, os resultados obtidos tornam possível um acompanhamento mais preciso das suas distribuições, e se necessário redistribui-las no interior do parque e na zona de amortecimento.

Sendo assim, os resultados do modelo podem ser utilizados para apoiar a gestão do Parque. Estudos incluindo mais variáveis antropogênicas, como o uso da terra, assim como outros fatores bióticos, como a faixa de dispersão e a competição, podem ser realizados para fortalecer o presente estudo.

Apesar do grande valor de uso identificado, principalmente Handroanthus impetiginosus (Mart. ex DC.), Caryocar coriaceum Wittm e Hymenaea courbaril L., nenhuma delas encontra-se na relação de espécies ameaçadas de extinção.

Vale ressaltar que o uso dessas espécies de grande utilidade e elevado valor comercial pelas comunidades do entorno do PNSC sofre restrições dentro do parque, por conta da fiscalização e controle dos órgãos ambientais, sendo possível observar a abundância das mesmas no interior dessa UC, em contraste com a sua relativa escassez em muitas áreas de entorno, existindo portanto uma relativa tensão entre a função de preservação exercida pelo parque e as comunidades vizinhas, o que exige o desenvolvimento de alternativas frente a essa situação nessas áreas de entorno, a exemplo de ações de Educação Ambiental e do desenvolvimento de projetos de reflorestamento para fins extrativistas.

\section{REFERÊNCIAS}

ALVES, A. A.; SALES, R. O.; NEIVA, J. N. M.; MEDEIROS, A. N.; BRAGA, A. P.; AZEVEDO, A. R. Degradabilidade ruminal in situ de vagens de faveira (Parkia platycephala Benth.) em diferentes tamanhos de partículas. Revista Brasileira de Medicina Veterinária e Zootecnia, v. 59, n. 4, p. 1045-1051, 2007. https://doi.org/10.1590/S010209352007000400034

AMARO, M. S.; MEDEIROS FILHO, S.; GUIMARÃES, R. M.; TEÓFILO, E. M. Morfologia de frutos, sementes e de plântulas de janaguba (Himatanthus drasticus (Mart.) Plumel - Apocynaceae). Revista Brasileira de Sementes, n. 28, v.1, 2006, p. 63-71, 2006. https://doi.org/10.1590/S0101-

31222006000100009

ANDERSON, R.P.; LEW, D. ; PETERSON, A.T. Evaluating predictive models of species' distributions: criteria for selecting optimal models. Ecological Modelling 162: 211-232. $2003 . \quad$ https://doi.org/10.1016/S03043800(02)00349-6

ARAÚJO, M. B.; CABEZA, M.; THUILLER, W.; HANNAH, L.; WILLIAMS, P. H. Would climate change drive species out of reserves? An assessment of existing reserve-selection methods. Global Change Biology, v. 10, n. 9, p. 1618-1626. 2004. https://doi.org/10.1111/j.13652486.2004.00828.x

AUSTIN, M. P. Spatial prediction of species distribution: an interface between ecological theory and statistical modelling. Ecological modelling, v. 157, n. 2-3, p. 101-118. 2002. https://doi.org/10.1016/S0304-3800(02)00205-3

BARROS, J. S.; FERREIRA, R. V.; PEDREIRA, A. J.; SCHOBBENHAUS, C. MINISTÉRIO DE MINAS E ENERGIA-SECRETARIA DE GEOLOGIA, MINERAÇÃO E TRANSFORMAÇÃO MINERAL. Projeto Geoparques GEOPARQUE SETE CIDADES - PEDRO II - PI Proposta. Serviço Geológico do Brasil - CPRM, 2014.

BOTREL, R. T.; OLIVEIRA FILHO, A. T; RODRIGUES, L. A.; CURI, N. Influência do solo e topografia sobre as variações da 
composição florística e estrutura da comunidade arbóreo-arbustivo de uma Floresta Estacional Semidecidual em Ingaí, - MG. Revista Brasileira de Botânica, v. 25, p. 195-213. 2002. https://doi.org/10.1590/S010084042002000200008

BRANDÃO, M.; LACA-BUENDIA, J. P.; MACEDO, J. F. Árvores nativas e exóticas do Estado de Minas Gerais. Belo Horizonte: EPAMIG, 528P. 2002.

CARVALHO, N. S. Propriedades microbiológicas do solo ao longo de um gradiente vegetacional de cerrado no Parque Nacional de Sete Cidade. 2016. $48 f$. Dissertação (Mestrado em Agronomia) Universidade Federal do Piauí, Teresina, 2016. CASTRO, A. A. J. F.; ARZABE, C.; CASTRO, N. M. C. F. Classificação e Caracterização dos Tipos Vegetacionais do Parque Nacional de Sete Cidades, Piauí, Brasil. Biodiversidade e ecótonos da região setentrional do Piauí. Teresina: EDUFPI, 2010. 208 p. (Série Desenvolvimento e Meio Ambiente, 5), 2010.

CASTRO, A. A. J. F.; SILVA, C. B.; FARIAS, R. R. S.; RAMOS NETO, M. B. Flora lenhosa em fragmentos de Floresta Estacional Semidecidual no Parque Nacional de Sete Cidades (PN7C), Piauí, Brasil. In: SantosFilho FS, Soares AFCL, Almeida Junior EB. (eds.) Biodiversidade do Piauí: pesquisas \& perspectivas. Curitiba, Editora CRV. p. 101119. 2013.

CONNERS, R. W.; Trivedi, M. M. and Harlow, C. A. "Segmentation of a High-Resolution Urban Scene Using Texture Operators", Computer Vision, Graphics, and Image Processing, Vol. 25, pp. 273-310. 1984. https://doi.org/10.1016/0734-189X(84)90197-X

COSTA, C. B.; CASTELLAN, D. C.; SOUZA, A. L.; SILVA, A. C. Boas Práticas de Manejo para o Extrativismo Sustentável do Jatobá. Brasília: Instituto Sociedade, População e Natureza, ISBN 978-85-63288-165. 76 p., 2015.

COSTA, W. S. Projeto: Prospecção do conhecimento científico de espécies florestais nativas (Convênio de Cooperação Técnica FAPEMIG / FUNARBE). Polo de Excelência em Florestas Universidade Federal de Viçosa, Viçosa-MG. Departamento de Engenharia Florestal Universidade Federal de Viçosa. Viçosa - MG. Março, 2011.

DURIGAN, G.; DIAS, H. C. de S. Abundância e diversidade da regeneração natural sob mata ciliar implantada. In: Congresso Florestal
Brasileiro, 6, Campos do Jordão. Anais. São Paulo: Sociedade Brasileira de Silvicultura, v.3, p.308-312. Publicado na Silvicultura, n.42, 1990.

FERREIRA, G. A.; SANTOS-VELOSO, V. R.; VELOSO-NAVES, R.; NASCIMENTO, J. L.; CHAVES, L. J. Biodiversidade de insetos em Pequizeiro (Caryocar brasiliense, Camb.) no cerrado do Estado de Goiás, Brasil. Agrociencia Uruguay, v. 13, n. 2, 2009.

FIELDING, A. H., BELL, J. F. A review of methods for the measurement of prediction errors in conservation presence/absence models. Environ. Conserv, 1997. https://doi.org/10.1017/S0376892997000088

FILGUEIRAS, T.S., BROCHADO, A.L., NOGUEIRA, P.E. \& GUALLA II, G.F. Caminhamento - Um método expedito para levantamentos florísticos qualitativos. In: Caderno de Geociência IBGE. 12: 39-43. 1994.

GIBSON, L., BARRETT, B., BURBIDGE, A. Lidando com a ausência incerta na modelagem de habitat: estudo de caso de uma terra rara no solo . Diversity and Distributions 13, 704713. 2007.

GOMES, S. O.; SILVA, R. A. O.; MARQUES, L. G. A.; SANTOS, M. R. M. C. Prospecção tecnológica: potencialidades de uso do pequizeiro (caryocar spp.). Revista GEINTEC - ISSN: 2237-0722. São Cristóvão/SE. Vol. 5/n. 1/ p.1617-1625 1617, 2015. https://doi.org/10.7198/S22370722201500010002

GUISAN, A.; BROENNIMANN, O.; ENGLER, R.; VUST, M.; YOCCOZ, N. G.; LEHMANN, A.; ZIMMERMANN, N. E. Using niche-based models to improve the sampling of rare species. Conservation Biology 20: 501-511. 2006. https://doi.org/10.1111/j.1523.

1739.2006.00354.x

GUISAN, A.; GRAHAM, C. H.; ELITH, J.; HUETTMANN, F.; The NCEAS Species Distribution Modelling Group. Sensitivity of predictive species distribution models to change in grain size. Diversity and Distributions 13: $332-340 . \quad 2007$. https://doi.org/10.1111/j.1472 4642.2007.00342.x

HARALICK, R. M.; SHANMUGAM, K.; DINSTEIN, I. "Textural Features for Image Classification", IEEE Trans. on Systems, Man and Cybernetics, Vol. SMC-3, pp. 610-621. 1973. https://doi.org/10.1109/TSMC.1973.4309314

HEIKKINEN, R. K.; LUOTO, M.; VIRKKALA, 
R.; PEARSON, R. G.; KÖRBER, J. H. BIOTIC interactions improve prediction of boreal bird distributions at macro-scales. Global Ecology and Biogeography 16: 754-763. 2007. https://doi.org/10.1111/j.1466-

8238.2007.00345.x

HIJMANS, R. J.; PHILLIPS, J.; LEATHWICK, J. E. Dismo: Species distribution modeling. $R$ package version 1.1-4. Date/Publication 2017-01-09 09:49:36.

https://cran.r-

project.org/web/packages/dismo/dismo.pdf. 2017.

HIJMANS, R. J.; GRAHAM, C.H. The ability of climate envelope models to predict the effect of climate change on species distributions. Global Change Biology 12: 2272-2281. 2006. https://doi.org/10.1111/j.13652486.2006.01256.x

HUETE, A. R. A. Soil-Adjusted Vegetation Index (SAVI). Remote Sensing of Environment, v. $25, \quad$ p. $295-309 . \quad 1988$ https://doi.org/10.1016/0034-4257(88)90106-X

JIMÉNEZ-VALVERDE， A.; LOBO， J. M. Threshold criteria for conversion of probability of species presence to either-or presenceabsence. Acta oecologica, v. 31, n. 3, p. 361369. 2007. https://doi.org/10.1016/j.actao.2007.02.001

JOLY, C. A.; FELIPPE, G. M.; DIETRICH, S. M. C.; CAMPOSTAKAKI, G. M. Physiology of germination and seed gel analysis in two populations of Magonia pubescens St. Hil. Revista Brasileira de Botânica, São Paulo, v. 3, p. 1-9. 1980.

KUMAR, S.; STOHLGREN, T. J. Maxent modeling for predicting suitable habitat for threatened and endangered tree Canacomyrica monticola in New Caledonia. J. Ecol. Nat. Environ. n. 1, p. 94-98. 2009.

LINDOSO, D. et al. Climate Change and Vulnerability to drought in the Semiarid: the case of smallholder farmers in the Brazilian northeast. In: SEROA DA MOTTA, R. et al. (Eds.). Climate change in Brazil: economic, social and regulatory aspects. Brasilia: IPEA, p. 235-256. 2011.

LIU, C., WHITE, M.; NEWELL, G. Selecting thresholds for the prediction of species occurrence with presence-only data. Journal of Biogeography n. 40, p.778-789. 2013. https://doi.org/10.1111/jbi.12058

LORENZI, H. Árvores brasileiras: manual de identificação e cultivo de plantas arbóreas nativas do Brasil. 4. ed. Nova Odessa, SP: Instituto Plantarum, 384p., 2013.
MARQUES, T. C. L. L. S.; SIQUEIRA, J. O.; MOREIRA, F. M. S. Crescimento de mudas de espécies arbóreas em solo contaminado com metais pesados. In: SIMPÓSIO MATOS, M. Q.; FELFILI, J.M. Florística, fitossociologia e diversidade da vegetação arbórea nas matas de galeria do Parque Nacional de Sete Cidades (PNSC), Piauí, Brasil (PNSC), Piauí, Brazil. Acta botânica brasílica, v. 24, n. 2, p. 483-496. 2010. https://doi.org/10.1590/S010233062010000200019

MATOS, M. Q.; FELFILI, J. M. Florística, fitossociologia e diversidade da vegetação arbórea nas matas de galeria do Parque Nacional de Sete Cidades (PNSC), Piauí, Brasil (PNSC), Piaú, Brazil. Acta botânica brasílica, v. 24, n. 2, p. 483-496, 2010. https://doi.org/10.1590/S0102-

33062010000200019

MELO-JUNIOR, A. F. de; CARVALHO, D.; POVOA, J. S. R.; BEARZOLI, E. Estrutura genética de populações naturais de pequizeiro (Caryocar brasiliense Camb). Scientia Forestalis, Piracicaba, v. 66, p. 56-65. 2004.

MESQUITA, M. R.; CASTRO, A. A. J. F. Florística e fitossociologia de uma área de cerrado marginal (Cerrado Baixo), Parque Nacional de Sete Cidades, Piauí. Publicações Avulsas em Conservação de Ecossistemas 15: 1-22. 2007. https://doi.org/10.18029/1809-0109/pace.n15p122

MORO, M. F.; CASTRO, A. S. F.; ARAÚJO, F. S. Composição florística e estrutura de um fragmento de vegetação savânica sobre os tabuleiros prélitorâneos na zona urbana de Fortaleza, Ceará. Rodriguésia 62:407-423 2011. https://doi.org/10.1590/21757860201162214

NACIONAL DE RECUPERAÇÃO DE ÁREAS DEGRADADAS, 3., Ouro Preto. Do substrato ao solo: trabalhos voluntários. Viçosa: Universidade Federal de Viçosa, 1997. p.429436. 1997.

NASCIMENTO, G. C. C.; CÓRDULA, E. B. L. Tradições, cultura e misticismo nas comunidades tradicionais: 'A antropólog'. Educação Pública, Rio de Janeiro, v. 16, n. 5, 2016.

NASCIMENTO, M. E. C.; NASCIMENTO, G. C. C.; CÓRDULA, E. B. L. Cultura e a oralidade nos contos tradicionais. Educação Pública, Rio de Janeiro, v.14, n. 20, 2014.

NUNES, G. M. Disponibilidade e uso de 
plantas medicinais na zona de amortecimento do Parque Nacional de Sete Cidades. Piauí - Brasil. Dissertação (mestrado). Universidade Federal da Paraíba, 2019.

OLIVEIRA, M. E. A; MARTINS, F. R.; CASTRO, A. A. J. F.; SANTOS, J. R. Classes de cobertura vegetal do Parque Nacional de Sete Cidades (transição campo-floresta) utilizando imagens TM/Landsat, NE do Brasil. Anais XIII Simpósio Brasileiro de Sensoriamento Remoto, Florianópolis, Brasil. 21-26 abril 2007, INPE, p. 1775-1783. 2007.

PELLETIER, J. D.; SWETNAM, T. L. Asymmetry of weathering-limited hillslopes: the importance of diurnal covariation in solar insolation and temperature. Earth Surface Processes and Landforms, v. 42, n. 9, p. 1408-1418. 2017.

https://doi.org/10.1002/esp.4136

PHILLIPS, S. J. Transferability, sample selection bias and background data in presence-only modeling: a response to Peterson et al. and Ecography, n.31, p.272-278, 2008. https://doi.org/10.1111/j.0906-7590.2008.5378.x

PHILLIPS, S. J.; ANDERSON, R. P.; SCHAPIRE, R.E. Maximum entropy modeling of species geographic distributions. Ecol. Model. n. 190, p. 231-259. 2006. https://doi.org/10.1016/j.ecolmodel.2005.03.026

RESENDE, M.; CURI, N.; REZENDE, S. B.; CORREAA, G. F. Pedologia: base para distinção de ambientes. 5. ed. Lavras, Editora da UFLA, 2007.

RIVERS, W. H. R. Medicine, magic and religion. London, Kegan Paul, 1924.

SALGADO-LABORIAU, M. L. S. A semente de Magonia pubescens St. Hil. - Morfologia e germinação. Anais da Academia Brasileira de Ciências, Rio de Janeiro, v. 45, n. 3, p. 1537, 1973.

SCALON, S. P. Q.; MUSSURY, R. M.; SCALON FILHO, H. Crescimento inicial de mudas de Bombacopsis glabra (Pasq.) A. Robyns sob diferentes níveis de sombreamento. Revista Árvore, v. 27, n. 6, p. 753-758. 2003. https://doi.org/10.1590/S0100-

67622003000600001

SILVA, H. H. G.; SILVA, I. G.; SANTOS, R. M. G.; RODRIGUES FILHO, E. ELIAS, C. N. Larvicidal activity of tannins isolated of Magonia puberscens St Hil (Sapindaceae) against Aedes aegypti (Diptera, Culicidae) Revista da Sociedade Brasileira de Medicina Tradicional, Ubatuba, v. 37, n 5, p. 396-399, 2004. https://doi.org/10.1590/S0037-
86822004000500005

SOBERÓN, J.; PETERSON, A.T. Interpretation of models of fundamental ecological niches and species distributional areas. Biodiversity Informatics 2 : 2005 . https://doi.org/10.17161/bi.v2i0.4

SOLÓRZANO, A. Análise Fitogeográfica do cerradão: conexões florísticas, padrões estruturais, relações ecológicas e modelagem de sua distribuição potencial. 2011. 115 f. Tese (Doutorado em Ecologia), Universidade de Brasília, Brasília, 2011.

SWETS, J. A. Measuring the accuracy of diagnostic systems. Science, n. 240, p.12851293. 1988.

https://doi.org/10.1126/science.3287615

THEOBALD, E. J.; ETTINGER A. K.; BURGESS, H. K.; DEBEY, L. B.; SCHMIDT, N. R. ; Wagner, H. E. C.; HILLERISLAMBERS,

J.; TEWKSBURY, J.; HARSCH, M. A.; PARRISH, J. K. Mudança global e soluções locais: Aproveitar o potencial não realizado da ciência cidadã para a biodiversidade pesquisa. Biol. Conserv. , 181, pp. 236-244. 2015. https://doi.org/10.1016/j.biocon.2014.10.021

VALADÃO, M. B. X.; MARIMON JR, B. H.; MORANDI, P. S.; REIS, S. M.; OLIVIERA, B.; OLIVEIRA, E. A.; MARIMON, B. S. Initial development and biomass patitioning of Physocalmma scaberrimum Pohl (Lythraceae) under different shading levels Scientia Forestalis, Piracicaba, v. 42, n. 101, p 129139. 2014.

WISZ, M. S.; HIJMANS, R. J.; LI, J.; PETERSON, A.T.; GRAHAM, C.H.; GUISAN, A.; NCEAS. Predicting species distributions working group. Effects of sample size on the performance of species distribution models. Diversity and Distributions 14: 763-773. 2008. https://doi.org/10.1111/j.1472 4642.2008.00482.x

YANG, X. Q.; KUSHWAHA, S. P. S.; SARAN, S.; $\mathrm{XU}$, J.; ROY, P. S. Maxent modeling for predicting the potential distribution of medicinal plant, Justicia adhatoda $L$. in Lesser Himalayan foothills. Ecol. Eng. n. 51, p.83-87, 2013. https://doi.org/10.1016/j.ecoleng.2012.12.004 